\title{
La flaveur des fromages \\ II. - ETUDE COMPARATIVE DE LA FRACTION VOLATILE NEUTRE DE DIVERS FROMAGES
}

\author{
par \\ M. GROUX* et M. MOINAS \\ Département de Recherche et Développement \\ Société d'Assistance Technique pour Produits Nestlé s.a. \\ Case postale, 1009, CH-1001 Lausanne \\ Directeur du Département R. \& D. : Professeur L. REY
}

\section{INTRODUCTION}

Dans un travail précédent [1], nous avons décrit une méthodologie originale d'isolement, d'enrichissement et d'analyse des constituants volatils des arômes de produits sensibles aux altérations (thermiques, microbiologiques, etc.) tels les produits laitiers. Les résultats d'analyses ont montré l'analogie qualitative qui existe entre les constituants volatils des arômes de Roquefort et de Camembert mûr. Nous avons pu aussi préciser l'importance des méthylcétones et des alcools primaires et secondaires pour la flaveur de ces deux fromages. Des tests organoleptiques effectués avec des arômes reconstitués et incorporés dans une base-fromage neutre (fromage fondu à base de Fontina jeune) ont permis de mettre en évidence l'importance de l'octène-1 ol-3 pour l'arôme de Camembert mûr.

Les bons résultats obtenus avec le Roquefort et le Camembert, selon la méthodologie déjà décrite, incitaient à étudier une gamme plus étendue de fromages. Parmi les 400 variétés de fromages cataloguées par G. Burkhalter [2], nous avons choisi, en plus du Roquefort et du Camembert, le Vacherin Mont-d'Or, le Cantal, le Gruyère, le Gouda et le Parmesan.

Nos travaux montrent que les arômes des fromages à pâte molle étudiés contiennent plus de méthylcétones que d'alcools primaires ou secondaires saturés. Les arômes des pâtes dures ou mi-dures au contraire sont généralement pauvres en méthylcétones et contiennent des quantités non-négligeables d'alcools primaires (surtout éthanol).

\footnotetext{
* Nouvelle adresse au 1-10-73 : Alpura-Koreco, 3510 Konolfingen (Suisse).
} 
Nous avons rencontré un cas intermédiaire : le Cantal dont l'arôme contient des quantités équivalentes de méthylcétones et d'alcools primaires.

Les résultats quantitatifs montrent que certains fromages sont très pauvres en constituants volatils (Vacherin Mont-d'Or, Gouda).

\section{MATERIEL ET METHODES}

L'isolement des constituants volatils des arômes, selon une technique de "stripping » sous pression réduite décrite par M. Moinas [3] a été effectué à partir d'une suspension d'environ $10 \mathrm{~kg}$ de fromage dans 20 à $25 \mathrm{~kg}$ d'eau bidistillée.

Le distillat aqueux obtenu (environ $5 \mathrm{~kg}$ ), bien que très dilué, avait dans tous les cas les caractéristiques olfactives typiques du fromage de départ. Cet isolat, cryoconcentré de 8 à 25 fois, a été ensuite extrait de manière continue par de l'éther éthylique distillé à l'aide d'un extracteur-concentrateur élaboré par H. Maarse [4]. La totalité des constituants volatils du cryoconcentrat $(100$ à $300 \mathrm{ml}$ selon les cas) était récupérée en solution dans 5 à $7 \mathrm{ml}$ d'éther éthylique. Pour les analyses en chromatographie gaz-liquide, certains des extraits éthérés ont été encore concentrés par évaporation du solvant sous conditions ménagées de température et dans un appareil décrit par $\mathbf{M}$. Moinas [3]. A ce sujet, nous avons vérifié à plusieurs reprises que l'éther éthylique utilisé pour l'extraction et concentré environ 100 fois dans cet appareil ne contenait pas de traces d'éthanol.

Les chromatogrammes sur Carbowax $20 \mathrm{M}$ (colonnes capillaires) obtenus selon des conditions opératoires identiques à celles appliquées précédemment [1] ont été dépouillés automatiquement selon un système de calculs des indices de Kovats mis au point par M. Moinas et J. Sotek [5]. En général, l'identification des constituants principaux des arômes a été confirmée par chromatographie en phase gazeuse et spectrométrie de masse combinées (appareil AEI MS 20, colonne Scott de Carbowax 20 M).

\section{RESULTATS ET DISCUSSION}

\section{A) Résultats qualitatifs et semi-quantitatifs}

Le tableau 1 regroupe les composés chimiques de nature méthylcétonique ou alcoolique (primaire ou secondaire) trouvés dans le 
TABLEAU 1. - Composition en constituants volatils neutres des arômes de divers fromages (en p. 100)

Fromages

\begin{tabular}{|c|c|c|c|c|c|c|c|}
\hline Composés chimiques & Roquefort & $\begin{array}{l}\text { Camembert } \\
\text { mûr }\end{array}$ & $\begin{array}{l}\text { Vacherin } \\
\text { Mont-d'Or }\end{array}$ & Cantal & Gruyère & Gouda & Parmesan \\
\hline Acétone & $1-5$ & tr. & $1-5$ & n.d. & $1-5$ & n.d. & n.d. \\
\hline Butanone & $1-5$ & n.d. & $1-5$ & $1-5$ & $1-5$ & $1-5$ & $1-5$ \\
\hline Pentanone-2 & $10-20$ & n.d. & $20-40$ & $20-40$ & $\operatorname{tr}$ & tr. & n.d. \\
\hline Heptanone-2 & $20-40$ & $1-5$ & $20-40$ & n.d. & $\operatorname{tr}$ & $10-20$ & $1-5$ \\
\hline Octanone-2 & n.d. & n.d. & n.d. & n.d. & n.d. & n.d. & n.d. \\
\hline Nonanone-2 & $10-20$ & $20-40$ & $1-5$ & n.d. & $\operatorname{tr}$ & n.d. & tr. \\
\hline Undécanone-2 & $\operatorname{tr}$ & $1-5$ & $1-5$ & n.d. & n.d. & n.d. & n.d. \\
\hline Ethanol & $1-5$ & tr. & $1-5$ & $20-40$ & env. 80 & $10-20$ & env. 80 \\
\hline Propanol & n.d. & n.d. & $1-5$ & n.d. & $1-5$ & n.d. & n.d. \\
\hline Butanol-2 & n.d. & n.d. & n.d. & n.d. & tr. & $20-40$ & tr. \\
\hline Pentanol-2 & $1-5$ & n.d. & $1-5$ & n.d. & $\operatorname{tr}$ & tr. & n.d. \\
\hline Heptanol-2 & $1-5$ & $10-20$ & $1-5$ & n.d. & tr. & tr. & tr. \\
\hline Nonanol-2 & tr. & $5-10$ & tr. & n.d. & n.d. & n.d. & n.d. \\
\hline Octène-1 ol-3 & tr. & $5-10$ & n.d. & n.d. & n.d. & n.d. & n.d. \\
\hline
\end{tabular}


Roquefort, le Camembert, le Vacherin Mont-d'Or, le Cantal, le Gruyère, le Gouda et le Parmesan.

Les constituants volatils trouvés dans le Roquefort correspondent bien à ceux mentionnés dans la littérature pour les fromages de type "bleu » [6]. En outre, ces résultats confirment ceux de D. P. Schwartz et O. W. Parks [7] qui mentionnent l'heptanone-2 comme constituant prépondérant. L'octanone-2 [8] et le propanol [9] mentionnés par d'autres auteurs n'ont pu être détectés dans les échantillons analysés.

La fraction volatile du Camembert mûr contient les constituants déjà connus tels que acétone, pentanone-2*, heptanone-2, nonanone-2, undécanone-2 [7]. Nous avons trouvé aussi la butanone*, l'éthanol, le propanol, le pentanol-2*, l'heptanol-2 et le nonanol-2 [1]. Le constituant présent en la plus grande quantité relative est ici la nonanone-2, confirmant en cela les travaux de D. P. Schwartz et O. W. Parks [7].

Le Vacherin Mont-d'Or, fromage local et saisonnier produit surtout dans le Jura Vaudois (Suisse) contient les mêmes composés que ceux trouvés dans le Roquefort à l'exception de l'octène1-01-3 qui n'a pas été identifiée. La fraction volatile neutre de ce fromage, contient surtout de la pentanone-2 et de l'heptanone-2.

Le Gruyère suisse analysé (temps d'affinage : 10 mois) contient les composés déjà connus tels que acétone, butanone, pentanone-2, heptanone-2, nonanone-2, éthanol et propanol $[9,10]$. L'éthanol représente environ 80 p. 100 des constituants volatils récupérés, ce qui confirme les résultats déjà publiés par J.E. Langler et al. [10]. Nos résultats mettent en évidence la présence de deux alcools secondaires jamais décrits : le pentanol-2 et l'heptanol-2. Par contre, l'undécanone-2 signalée par J.E. Langler [11] n'a pas été détectée dans l'échantillon analysé.

La fraction volatile du Gouda, contient des constituants déjà décrits par certains auteurs [12], tels que butanone, pentanone-2 et heptanone-2. Les résultats du tableau 1 indiquent la présence des constituants nouveaux suivants : éthanol, butanol-2, pentanol-2, heptanol-2. Par contre, l'acétone et la nonanone-2 trouvés par D. V. Anderson et E.A. Day [13] n'ont pas été détectés dans l'échantillon analysé. Le butanol-2 représente environ $1 / 3$ des constituants volatils totaux récupérés.

La fraction volatile isolée du Cantal est assez simple et se limite pratiquement à la butanone, la pentanone-2 et l'éthanol. Ces deux derniers sont présents en quantités relativement équivalentes. Les composés volatils de nature cétonique et alcoolique de ce fromage ne semblent jamais avoir été décrits jusqu'à présent.

Comme dans le Gruyère, l'éthanol est le constituant majeur de la fraction volatile isolée du Parmesan. On trouve en plus la butanone. l'heptanone-2, la nonanone-2 et l'heptanol-2.

\footnotetext{
* Détectés seulement dans le Camembert jeune [1].
} 


\section{B) Résultats quantitatifs}

Les constituants volatils des sept fromages étudiés ont été isolés dans les mêmes conditions. Il est raisonnable de penser que la rétention de ces constituants lors de l'entraînement à l'argon sous pression réduite ne fut pas très différente d'un fromage à un autre. La concentration en constituants volatils dans l'extrait éthéré a pu être déterminée par les valeurs d'intégration des surfaces des pics chromatographiques. Les taux de cryoconcentration des solutions aqueuses de constituants volatils ainsi isolés étant connus, il devenait possible de calculer les quantités absolues des constituants volatils récupérées par kg de chaque fromage par entraînement à l'argon sous pression réduite. En fixant arbitrairement la quantité absolue récupérée du Camembert égale à 1 , on obtient les données suivantes :

\section{TABLEAU 2}

Quantités relatives de constituants volatils de divers fromages récupérables par entraînement à l'argon sous pression réduite

\begin{tabular}{l|c}
\hline \multicolumn{1}{c|}{ Fromage } & Quantité relative \\
\hline Camembert mûr & 1,0000 \\
Roquefort & 0,3750 \\
Vacherin Mont-d'Or mûr & 0,0063 \\
Gruyère & 0,0220 \\
Parmesan & 0,0210 \\
Gouda & 0,0048 \\
Cantal & 0,0950 \\
\end{tabular}

Les quantités de constituants volatils récupérés sont très différentes d'un fromage à un autre. En particulier, celles isolées du Vacherin Mont-d'Or et du Gouda sont très faibles. Il est particulièrement intéressant de noter que ces quantités sont du même ordre de grandeur pour le Gruyère et le Parmesan.

Comme l'indique le tableau 1, les constituants volatils des arômes des fromages étudiés appartiennent en grande partie à des séries homologues : méthylcétones, alcools primaires, alcools secondaires saturés et pour le Camembert et le Roquefort, un alcool secondaire insaturé (octène-1 ol-3). Si l'on combine les valeurs des tableaux 1 et 2 
et que l'on fixe arbitrairement égale à 100 la quantité totale de méthylcétones récupérées du Camembert mûr par entraînement à l'argon sous pression réduite, on remarque (fig. 1) :

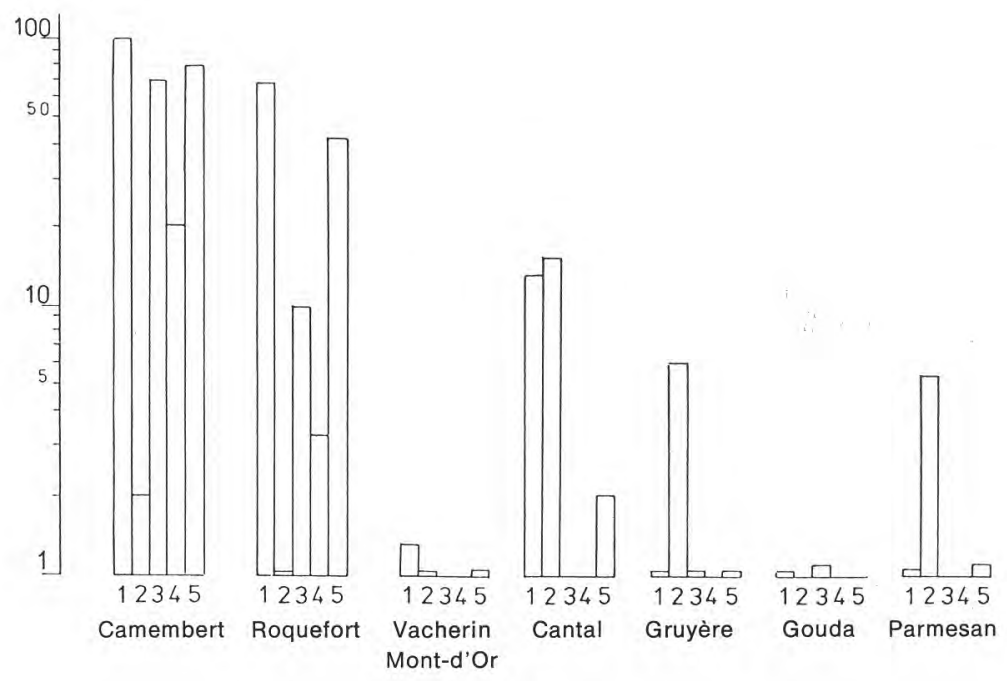

fig. 1

Quantités relatives* de méthylcétones et d'alcools primaires et secondaires récupérés de divers fromages par entraînement à l'argon sous pression réduite

( ${ }^{*}$ les méthylcétones du Camembert $\left.=100\right)$

1 : méthylcétones.

2 : alcools primaires saturés.

3 : alcools secondaires saturés.

4 : alcools secondaires non-saturés (octène-1 ol-3).

5 : constituants non-cétoniques et non-alcooliques.

1) les méthylcétones sont présentes en quantités équivalentes dans le Roquefort et le Camembert. Dans le Cantal, elles se trouvent en quantités inférieures mais non-négligeables. Par contre, elles ne sont présentes qu'à l'état de traces dans le Vacherin Mont-d'Or, le Gruyère, le Gouda et le Parmesan ;

2) les alcools primaires, surtout l'éthanol, sont peu abondants dans les pâtes molles (Camembert, Roquefort et Vacherin Mont-d'Or). Par contre, l'éthanol apparaît en quantités importantes dans les pâtes dures (Cantal, Gruyère, Parmesan). Il n'apparaît qu'à l'état de traces dans le Gouda ; 
3) les alcools secondaires saturés sont peu abondants dans les fromages autres que Camembert mûr et Roquefort. On notera tout particulièrement la relative richesse du Camembert en ces composés ;

4) l'octène-1 ol-3 est présent uniquement dans le Camembert et le Roquefort. Dans ce dernier, il est moins abondant que dans le Camembert. Comme déjà signalé précédemment [1], il est possible que cet alcool insaturé ait son importance pour la flaveur du Camembert mûr ;

5) enfin, les quantités de composés volatils neutres autres que méthylcétones et alcools (primaires et secondaires) sont relativement faibles sauf pour le Camembert et le Roquefort. Parmi ceux-ci, on peut citer des composés phénoliques, des lactones et des hydrocarbures supérieurs [1].

\section{CONCLUSIONS}

La figure 1 montre la grande analogie existant entre les fractions volatiles des arômes de Camembert mûr et de Roquefort. La différence la plus remarquable se situe au niveau des quantités relatives d'alcools secondaires (saturés et insaturés). Toutefois, selon nos résultats précédents [1], il semble que seule la différence en octène-1 o1-3 soit importante pour la flaveur de ces deux fromages.

La fraction volatile de l'arôme du Vacherin Mont-d'Or, même si elle est très complexe (tab. 1), est quantitativement très faible. Les quantités de méthylcétones dans ce fromage sont proches de leurs seuils de perception dans le lait soit $0,5 \mathrm{ppm}$ pour la pentanone-2 [14] et $0,7 \mathrm{ppm}$ pour l'heptanone-2 [15]. Il en est de même pour les autres constituants volatils détectés. Dans ces conditions, il paraît évident que la flaveur typique du Vacherin Mont-d'Or est à attribuer à des constituants d'une autre nature et qui restent à déterminer.

Un autre fromage dont la fraction volatile est faible est le Gouda. Ici, les quantités de constituants méthylcétoniques et alcooliques récupérées sont inférieures à leurs seuils de perception respectifs.

L'analogie entre les fractions volatiles du Gruyère et du Parmesan est assez inattendue. Le composé principal dans les deux cas est l'éthanol. Les quantités de méthylcétones récupérées sont là aussi inférieures à leurs seuils de perception. Selon J. E. Langler et al. [10] la flaveur typique du Gruyère serait due à la présence combinée et équilibrée de constituants volatils, d'acides gras libres et d'acides aminés. T. Kristoffersen [16] au contraire, pense que les composés soufrés (mercaptans) seraient des constituants importants de la flaveur.

Enfin, le Cantal semble être un cas très particulier. Sa fraction volatile contient, pour une pâte dure, des quantités importantes de 
méthylcétones (surtout pentanone-2). Par ailleurs, elle contient 2 fois plus d'éthanol que celle du Gruyère et du Parmesan. La quantité de pentanone-2 récupérée est largement supérieure au seuil de perception de cette méthylcétone. Il est raisonnable de penser que ce constituant joue un rôle important pour la flaveur de ce fromage. Toutefois, l'aromatisation d'une base fromage neutre (par ex. Fontina jeune) avec un mélange de pentanone-2 et d'éthanol dans les proportions trouvées n'a pas permis de reproduire la flaveur typique du Cantal.

\section{R és u m é}

La méthodologie d'analyse décrite précédemment [1] a été utilisée pour l'étude comparative des constituants volatils de sept fromages différents : Camembert, Roquefort, Vacherin Mont-d'Or, Cantal, Gruyère, Gouda et Parmesan.

Les quantités de constituants volatils récupérés varient énormément selon les fromages. Le Camembert et le Roquefort sont très riches en constituants volatils en comparaison du Vacherin Mont-d'Or, du Gouda, du Gruyère et du Parmesan. Le Cantal est relativement plus riche en ces constituants que les quatre derniers cités.

Alors que le Camembert, le Roquefort et le Cantal contiennent des quantités importantes de méthylcétones, celles-ci sont peu abondantes dans les autres fromages étudiés. Le Cantal, le Gruyère et le Parmesan contiennent des quantités notables d'alcools primaires (surtout d'éthanol). Par contre le Camembert et le Roquefort sont relativement pauvres en ces constituants. Les alcools secondaires saturés et insaturés (octène-1 ol-3) sont surtout présents dans les pâtes molles (Camembert et Roquefort). Ils font presque défaut dans les autres fromages.

L'importance organoleptique des constituants trouvés est discutée pour chaque fromage.

\section{S u m m a r y}

The method of analysis, described in another paper [1] has been used for a comparative study on the volatile components of seven cheeses : Camembert, Roquefort, Vacherin Mont-d'Or, Cantal, Gruyère, Gouda and Parmesan.

The total quantities of volatile constituents are very variable from one cheese to another. For example, Camembert and Roquefort contain the largest quantities while Vacherin Mont-d'Or, Gouda and also Gruyère and Parmesan contain little quantities of volatile compounds. Cantal is relatively rich in these compounds too. 
Large quantities of methylketones are found in Camembert, Roquefort and Cantal. The other cheeses do not contain them in appreciable amounts. Cantal, Gruyère and Parmesan contain important quantities of primary alcohols (mainly ethanol). These are only found in minor quantities in Camembert and Roquefort. The secondary alcohols, saturated and unsaturated (1 oct-en- 3 ol) are found mainly in Camembert and Roquefort.

The organoleptic significance of the volatile constituents found is discussed for each cheese.

\section{Remerciements}

Nous remercions Mlles D. Keller, S. Ledermann et E. Schmidt et M. $K$. Rolli et ses collaborateurs pour leur aide technique.

L'aide de M. J. Sotek pour le dépouillement des chromatogrammes par ordinateur a été tout particulièrement appréciée.

Reçu pour publication en septembre 1973.

\section{Références}

[1] Moinas (M.), Groux (M.) et Horman (I.) (1973). - Le Lait, 53, (529-530), 601.

[2] BuRKalter (G.) (1971). - Bulletin annuel de la Fédération Internationale de Laiterie, Partie IV.

[3] Moinas (M.) (1973). - Mitt. Lebensmittelunters, u. Hyg., 64, (1), 60.

[4] MaArse (H.) (1971). - Thèse, Université de Groningue (Pays-Bas).

[5] Moinas (M.) et Sotek (J.). - En préparation.

[6] Patton (S.) (1950). - J. Dairy Sci., 33, 680.

[7] Schwartz (D. P.) and Parks (O. W.) (1963). - J. Dairy Sci., 46, 1136.

[8] Bavisotto (V.S.) (1962). - Brevet U.S. no 3034902.

[9] Burger (A. M.) (1968). - Die natürlichen und künstlichen Aromen, Dr A. Hütig Verlag, Heidelberg (R.F.A.).

[10] Langler (J. E.), Libbey (L. N.) and Day (E. A.) (1967). - J. Agr. Food Chem., $15,386$.

[11] Langler (J. E.) (1968). - Thèse, Université de l'Etat d'Oregon (U.S.A.).

[12] Nakanishi (J.) and Nakazawa (Y.) (1966). - Int. Dairy Congr. Proc, 17 th., Munich, 4, 307.

[13] Anderson (D. F.) and Day (E. A.) (1966). - J. Agr. Food Chem., 14, 241.

[14] Honkanen (E.), Karvonen (P.) and Virtanen (A.) (1964). - Acta Chem. Scand., 18, 612.

[15] Kinsella (J. E.) (1969). - Chem. Ind., 36.

[16] Kristoffersen (T.) (1967). - J. Dairy Sci., 50, 279. 